\title{
645 V Quasi-Vertical GaN Power Transistors on silicon substrates
}

\author{
Chao Liu, Riyaz Abdul Khadar, and Elison Matioli \\ École Polytechnique Fédérale de Lausanne (EPFL) \\ CH-1015 Lausanne, Switzerland \\ chao.liu@epfl.ch, elison.matioli@epfl.ch
}

\begin{abstract}
In this paper, we present GaN-on-Si vertical transistors consisting of a $6.7 \mu \mathrm{m}$ thick n-p-n heterostructure grown on 6-inch silicon substrates by MOCVD. The fabricated vertical trench gate MOSFETs exhibited E-mode operation with a threshold voltage of $3.3 \mathrm{~V}$ and an on/off ratio of over $10^{8}$. A specific on-resistance of $6.8 \mathrm{~m} \Omega \cdot \mathrm{cm}^{2}$ and a high off-state breakdown voltage of $645 \mathrm{~V}$ were achieved. These results show the great potential of the GaN-on-Si platform for the next generation of cost-effective power electronics.
\end{abstract}

Keywords-GaN; vertical transistors; GaN-on-Si; power; semiconductor; devices; MOSFETS

\section{INTRODUCTION}

GaN-based semiconductors and devices have progressed rapidly in the past decades. The dominant III-nitride power devices with a high breakdown voltage are lateral $\mathrm{AlGaN} / \mathrm{GaN}$ high electron mobility transistors (HEMTs), which have the distinct advantages of polarization charges at the heterointerface to produce a high-density and high-mobility two-dimensional electron gas (2DEG) that effectively reduces the on-resistance and increases the switching speeds of highvoltage devices. However, lateral GaN HEMTs have some limitations for power applications. For example, the threshold voltage $\left(\mathrm{V}_{\mathrm{TH}}\right)$ of most GaN HEMTs is not high enough for failsafe operation in power systems. In addition, the lateral topology is not suitable for high-voltage and high-current applications. Substantial gate-to-drain spacing is required to achieve high breakdown voltages, which increases the chip size and reduces the effective device current density.

Vertical topology, on the other hand, provides a feasible solution for high-power-density devices. By increasing the thickness of the drift layer, the breakdown voltage can be increased without sacrificing the device size. There have been several reports of high performance GaN-based vertical transistors on freestanding GaN substrates [1-13]. However, the high cost and small available size of bulk GaN substrates limit the wide-spread commercial adoption of vertical power devices on bulk GaN. The GaN-on-Si platform offers a costeffective alternative for vertical GaN power devices, due to its large-scale availability, low cost, and a mature fabrication technology [14-20].

In this work, we demonstrate quasi-vertical GaN-on-Si vertical transistors based on a $6.7 \mu$ m-thick n-p-n heterostructure grown on 6-inch silicon substrates. The fabricated vertical trench gate MOSFETs exhibited E-mode

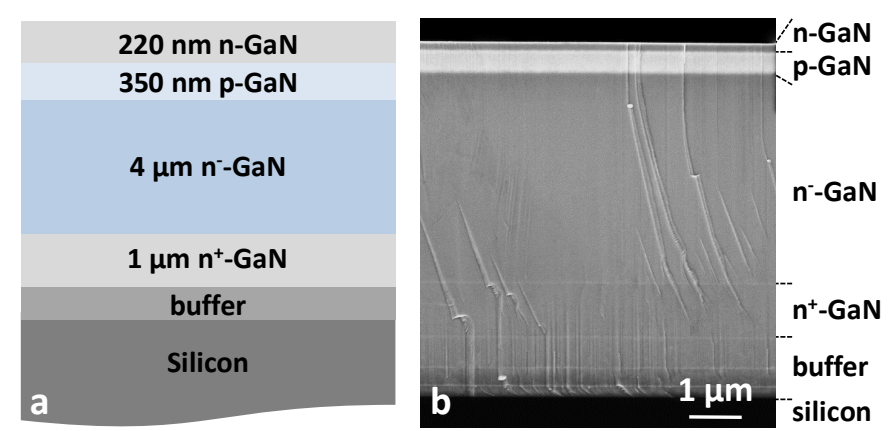

Fig. 1. Cross-sectional (a) schematic, and (b) SEM image of the as-grown n$\mathrm{p}$-n heterostructure on 6-inch silicon substrates.

operation with a $\mathrm{V}_{\mathrm{TH}}$ of $3.3 \mathrm{~V}$ and an on/off ratio of over $10^{8}$. A specific on-resistance $\left(\mathrm{R}_{\mathrm{ON}, \mathrm{SP}}\right)$ of $6.8 \mathrm{~m} \Omega \cdot \mathrm{cm}^{2}$ and a high offstate breakdown voltage of $645 \mathrm{~V}$ were achieved. The excellent performance can be attributed to the optimized $4 \mu \mathrm{m}$ drift layer. This excellent performance reveals the great potential of GaNon-Si to serve as a platform for future power electronic applications.

\section{DEVICE STRUCTURE AND FABRICATION}

The n-p-n heterostructure used in this work was grown on 6-inch Si (111) substrates by metal organic chemical vapor deposition system. Fig. 1 (a) presents the schematic structure of the n-p-n structure on silicon substrates. From bottom to top, the n-p-n structure consisted of a $1.1 \mu$ m-thick buffer layer, a 1 $\mu$ m-thick n-type GaN layer (Si dopant concentration $\sim 1 \times 10^{19}$ $\mathrm{cm}^{-3}$ ), a $4 \mu \mathrm{m}$-thick n-type GaN layer (Si dopant concentration $\sim 2 \times 10^{16} \mathrm{~cm}^{-3}$ ), a $350 \mathrm{~nm}$-thick p-type GaN layer (Mg dopant concentration $\sim 4 \times 10^{19} \mathrm{~cm}^{-3}$ ), a $200 \mathrm{~nm}$-thick n-type GaN layer (Si dopant concentration $\sim 5 \times 10^{18} \mathrm{~cm}^{-3}$ ), and a $20 \mathrm{~nm}$ thick n-type GaN layer (Si dopant concentration $\sim 1 \times 10^{19} \mathrm{~cm}^{-}$ ${ }^{3}$ ). Cross-sectional SEM image in Fig. 1 (b) shows a total epitaxial thickness of $6.7 \mu \mathrm{m}$, with an abrupt junction interface. A thick GaN drift layer with high crystalline quality is required to achieve high breakdown characteristics for $\mathrm{GaN}$ vertical devices. A $4 \mu \mathrm{m}$-thick continuously grown $\mathrm{GaN}$ drift layer on silicon was achieved with a low dislocation density of $3 \times 10^{8}$ $\mathrm{cm}^{-2}$, without the use of any interlayer which would significantly degrade the $\mathrm{R}_{\mathrm{ON}, \mathrm{SP}}$. After growth, the wafer bowing was $\mathrm{X} \sim-57 \mu \mathrm{m}, \mathrm{Y} \sim-45 \mu \mathrm{m}$, indicating excellent strain management of the thick GaN growth.

A schematic process flow is depicted in Fig. 2. The device fabrication started with a plasma-based dry etching process of

This work was supported by the European Research Council under the European Union's H2020 programme/ERC Grant Agreement 679425.

(C) 2018 IEEE. Personal use of this material is permitted. Permission from IEEE must be obtained for all other uses, in any current or future media, including reprinting/republishing this material for advertising or promotional purposes, creating new collective works, for resale or redistribution to servers or lists, or reuse of any copyrighted component of this work in other works. 

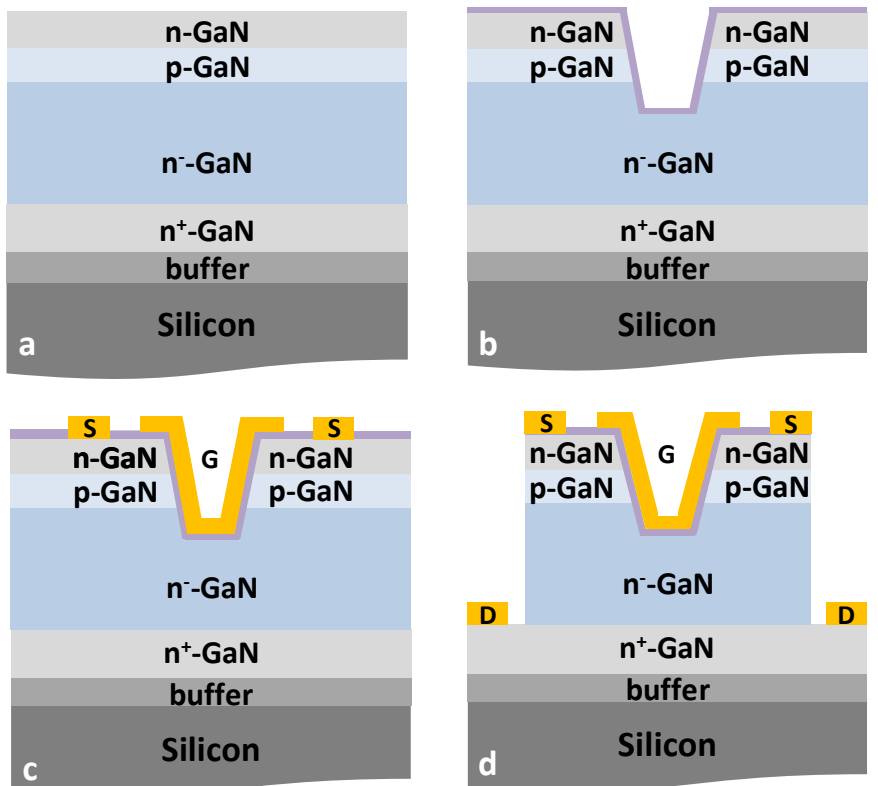

Fig. 2. Schematic process flow for GaN-on-Si vertical MOSFETs, (a) asgrown n-p-n structure on silicon, (b) trench etching and ALD $\mathrm{SiO}_{2}$ deposition, (c) source contact opening, and gate/source metal deposition, (d) deep etching and drain metal deposition.

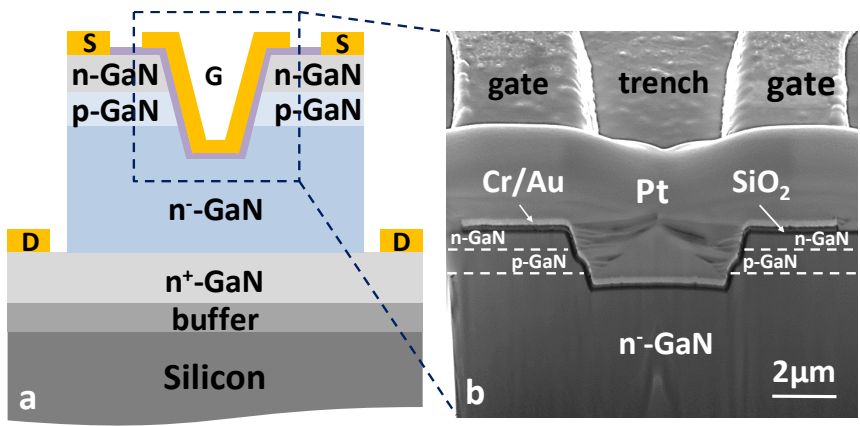

Fig. 3. Cross-sectional (a) schematic, and (b) SEM image of the fabricated quasi-vertical trench gate MOSFETs on Si substrate.

$\mathrm{GaN}$ to form the trench structures for the vertical gate. The sample was then treated with a Tetra Methyl Ammonium Hydroxide (TMAH) solution. A rapid thermal annealing was performed at $850^{\circ} \mathrm{C}$ for $20 \mathrm{~min}$ in a $\mathrm{N}_{2}$ ambient to activate the p-type GaN. Subsequently, a $\mathrm{SiO}_{2}$ gate dielectric for the vertical MOSFETs was deposited on the top surface and trenches by atomic layer deposition. After opening contact holes, a double-layer metal stack of $\mathrm{Cr} / \mathrm{Au}$ was evaporated to form both the source and gate electrodes for the vertical MOSFETs. Finally, a $5 \mu \mathrm{m}$-deep etching was performed using a ICP etch, followed by the evaporation of drain electrodes. More Details of fabrication can be found in [21].

\section{RESULTS AND DISCUSSION}

Fig. 3 (a) and (b) presents the cross-sectional schematic and SEM image of the GaN-based vertical trench gate MOSFET on silicon substrates. A $1.6 \mu \mathrm{m}$ deep gate trench can be observed by FIB-SEM. The trench sidewalls were inclined by $13.2^{\circ}$ from the c-axis, resulting in a trench width of $4.0 \mu \mathrm{m}$ and $5.5 \mu \mathrm{m}$ at the bottom and the top, respectively. Due to the erosion of the edge of the $\mathrm{SiO}_{2}$ hard mask during dry etching, a small kink
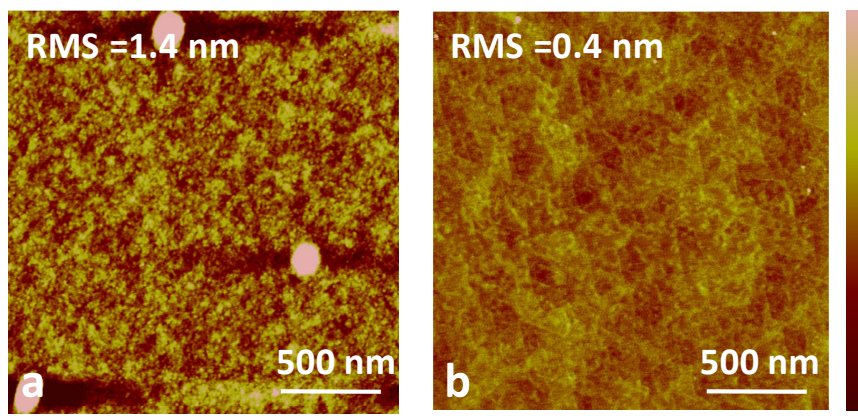

Fig. 4. AFM images of the ICP etched surface (a) without TMAH treatment, and (b) with TMAH treatment.
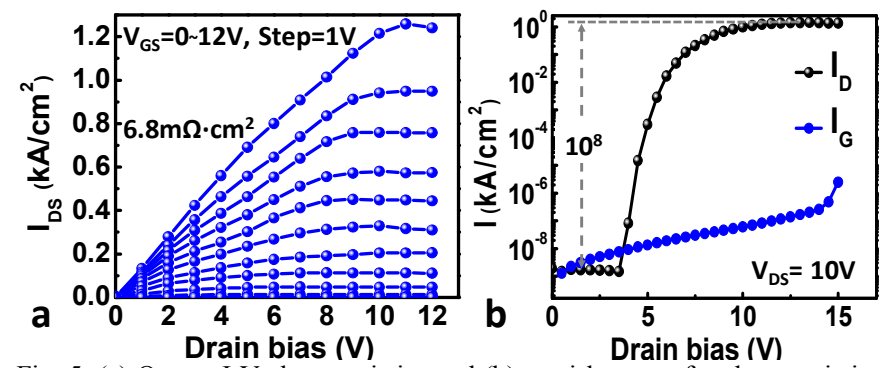

Fig. 5. (a) Output I-V characteristics and (b) semi-log transfer characteristics of the vertical trench gate MOSFETs on silicon substrate.
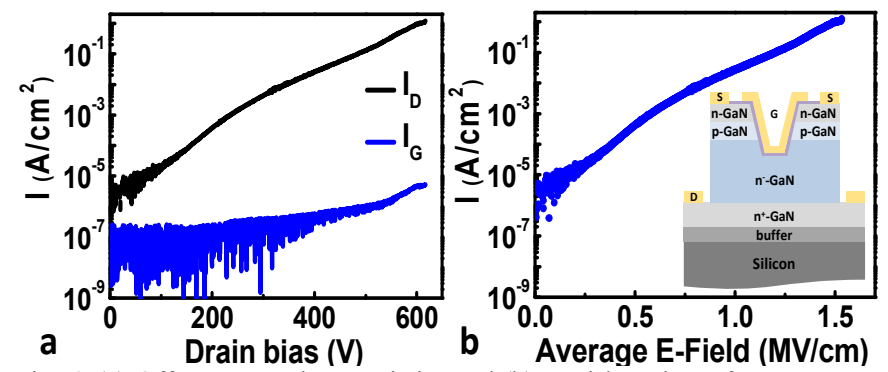

Fig. 6. (a) Off-state I-V characteristics and (b) semi-log plots of I versus $E$ at $\mathrm{V}_{\mathrm{GS}}=0 \mathrm{~V}$ of the vertical trench gate MOSFETs on silicon substrate.

was formed at the middle position of the sidewall. The sidewall and bottom of the gate trench were well covered by $\mathrm{SiO}_{2}$ gate dielectric and $\mathrm{Cr} / \mathrm{Au}$ gate metal, which is crucial to obtain a low leakage and low on-resistance vertical trench gate MOSFET.

It has been reported that TMAH wet etch is effective in removing damage from the sidewall and bottom of the dryetched gate trench, which can improve the conductivity of the inversion channel and eliminate the high electric field peaks at the bottom of the gate region [22]. To investigate the effect of TMAH treatment on the dry etched GaN surface, ICP etching was carried out to remove the top $1 \mu \mathrm{m}$ layer from the asgrown $n-p-n$ structure, followed by TMAH treatment at $85^{\circ} \mathrm{C}$ for 60 minutes. Fig. 4 compares the AFM images of the dry etched sample (a) before and (b) after TMAH treatment in an area of $2 \times 2 \mu^{2}$. Large dots can be observed on the ICP exposed $\mathrm{n}^{-} \mathrm{-GaN}$ surface, which might be caused by the unoptimized ICP etching conditions. The surface morphology of the TMAH treated sample is dramatically improved with a root mean squire (RMS) roughness of $0.4 \mathrm{~nm}$ from $1.4 \mathrm{~nm}$ before treatment, indicating that TMAH is effective in removing the dry etching damages and smoothing the etched surface.

(C) 2018 IEEE. Personal use of this material is permitted. Permission from IEEE must be obtained for all other uses, in any current or future media, including reprinting/republishing this material for advertising or promotional purposes, creating new collective works, for resale or redistribution to servers or lists, or reuse of any copyrighted component of this work in other works. 

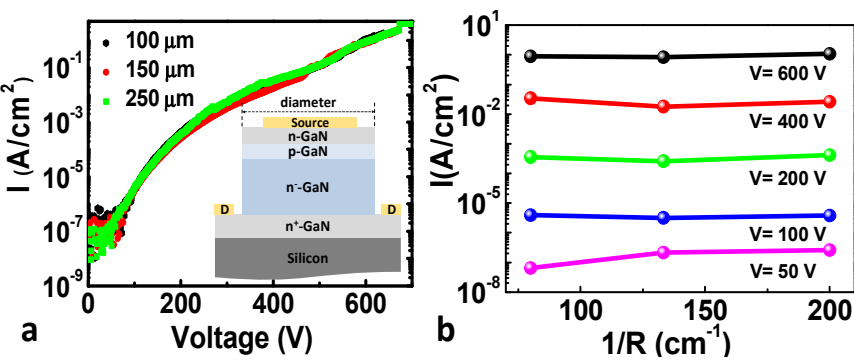

Fig. 7. (a) Two-terminal I-V characteristics of the as-grown n-p-n structure and (b) leakage current density of the two-terminal circular n-p-n test structure with different mesa radius $\mathrm{R}$.

Fig. 5 (a) shows the output I-V characteristics $\left(\mathrm{I}_{\mathrm{D}}-\mathrm{V}_{\mathrm{DS}}\right)$ of the fabricated vertical MOSFETs. The $\mathrm{R}_{\mathrm{ON}, \mathrm{SP}}$ calculated from the linear region using the trench area was $6.8 \mathrm{~m} \Omega \cdot \mathrm{cm}^{2}$, which could be further reduced by post-trench-etching treatment using a higher concentration of TMAH. At $\mathrm{V}_{\mathrm{GS}}=12 \mathrm{~V}$ and $\mathrm{V}_{\mathrm{DS}}=11 \mathrm{~V}$, the drain current density of $1.3 \mathrm{kA} / \mathrm{cm}^{2}$ was obtained, with good saturation behavior. Fig. 5 (b) shows the transfer I-V characteristics $\left(\mathrm{I}_{\mathrm{D}}-\mathrm{V}_{\mathrm{GS}}\right)$ of the vertical MOSFETs at a drain-source voltage of $10 \mathrm{~V}$. The fabricated vertical MOSFETs showed a current on/off ratio of over $10^{8}$ and a sub-threshold swing (SS) of $250 \mathrm{mV} / \mathrm{dec}$. A slight increase in gate leakage at $\mathrm{V}_{\mathrm{GS}}=15 \mathrm{~V}$ can be observed, which indicates that the trench etching and gate dielectric deposition can be further improved. A $\mathrm{V}_{\mathrm{TH}}$ of $3.3 \mathrm{~V}$ can be extracted from semilog scale plots. The high $\mathrm{V}_{\mathrm{TH}}$ is highly desirable to guarantee a fail-safe operation in high power applications. The off-state leakage current level was below $10^{-8} \mathrm{kA} / \mathrm{cm}^{2}$, indicating effective current blocking by the n-p-n heterostructure.

Fig. 6 (a) shows the off-state I-V characteristics measured at $\mathrm{V}_{\mathrm{GS}}=0 \mathrm{~V}$ for the fabricated trench gate MOSFETs. A large hard breakdown voltage ( $V_{\text {Boff }}$ ) of $645 \mathrm{~V}$ was observed at 2.8 $\mathrm{A} / \mathrm{cm}^{2}$ for the vertical MOSFETs. The breakdown was destructive and mainly observed at the mesa edge regions, which could be improved with edge termination technologies. However, the performance observed even without edge termination is remarkable, which shows the huge potential for $\mathrm{GaN}$ on $\mathrm{Si}$ vertical transistors. The gate current below $10^{-5}$ $\mathrm{A} / \mathrm{cm}^{2}$, implying that the drain leakage current originates from a leakage current between the source and the drain contacts. The off-state leakage mechanism was further identified by studying the correlation between leakage current density I and E field. A nearly linear relationship of $\ln (\mathrm{I}) \propto \mathrm{E}$ was found for the fabricated vertical MOSFETs, as plotted in Fig. 6 (b). Referring to [23], the $\ln$ (I) $\propto$ E linearity indicates that the leakage mechanism is dominated by variable-range hopping for the vertical MOSFETs in this work.

A two terminal breakdown test for the as-grown n-p-n structure was also performed. As shown in Fig. 7 (a), the breakdown voltage of the $n-p-n$ structure $\left(V_{\mathrm{BR}}\right)$ is $\sim 679 \mathrm{~V}$, which is consistent with the MOSFET breakdown voltage. The measured leakage current mainly flows through the n-p-n heterostructures, instead of the etched surfaces. To test this, we have measured the leakage current from two-terminal circular $n-p-n$ test structures with different mesa radius $(\mathrm{R}=$

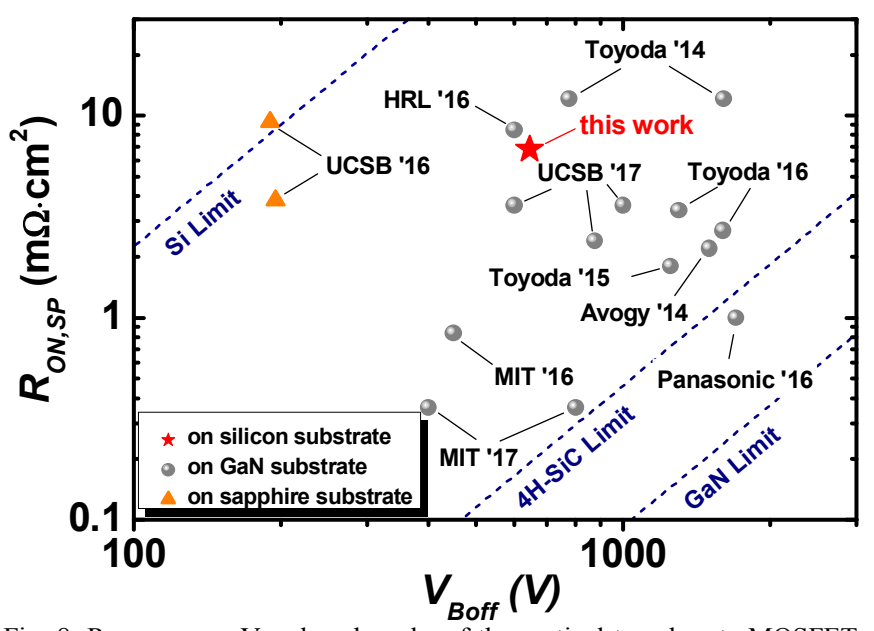

Fig. 8. $\mathrm{R}_{\mathrm{ON}, \mathrm{SP}}$ versus $\mathrm{V}_{\mathrm{Boff}}$ benchmarks of the vertical trench gate MOSFETs on silicon substrates with state-of-the-art E-mode vertical transistors on sapphire and GaN substrates.

$50 \mu \mathrm{m}, 75 \mu \mathrm{m}$, and $125 \mu \mathrm{m}$ ), and found the exact same leakage current density with different radius (no dependence of leakage current density on the mesa periphery in Fig. 7 (b)). This indicates that the device leakage current is mainly through the heterostructure instead of the etched sidewall.

Our GaN-on-Si vertical transistors were benchmarked in Fig. 8 against state-of-the-art E-mode vertical GaN transistors on $\mathrm{GaN}$, and sapphire substrates as a function of $\mathrm{V}_{\text {Boff. With a }}$

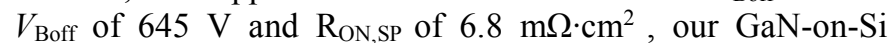
vertical transistors showed a very good Baliga figure-of-merit (FOM) of $61 \mathrm{MW} / \mathrm{cm}^{2}$. The FOM can be significantly improved by designing field plate and edge termination technologies as well as optimizing the growth and device fabrication.

\section{CONCLUSION}

In summary, GaN-on-Si vertical MOSFETs are demonstrated on 6-inch silicon substrates. The fabricated vertical MOSFETs exhibited a threshold voltage of $3.3 \mathrm{~V}$ and a specific on-resistance of $6.8 \mathrm{~m} \Omega \cdot \mathrm{cm}^{2}$. An off-state breakdown voltage of $645 \mathrm{~V}$ was achieved, thanks to the highquality 4- $\mu \mathrm{m}$-thick GaN drift layer. These results make this GaN-on-Si vertical MOSFETs very promising for costeffective and high-performance power electronics applications.

\section{ACKNOWLEDGMENT}

We would like to thank the staff at CMi and ICMP cleanrooms at EPFL for technical support and advice. We would also like to thank Dr. Kai Cheng from Enkris Semiconductor, Inc for the collaboration on the growth of high-quality customized wafers.

\section{REFERENCES}

[1] H. Otake, S. Egami, H. Ohta, Y. Nanishi, and H. Takasu, "GaN-based trench gate metal oxide semiconductor field effect transistors with over $100 \mathrm{~cm}^{2} /(\mathrm{Vs})$ channel mobility,” Jpn. J. Appl. Phys., vol. 46, no. 25, pp. L599-L601, Jul 2007.

(C) 2018 IEEE. Personal use of this material is permitted. Permission from IEEE must be obtained for all other uses, in any current or future media, including reprinting/republishing this material for advertising or promotional purposes, creating new collective works, for resale or redistribution to servers or lists, or reuse of any copyrighted component of this work in other works. 
[2] H. Otake, K. Chikamatsu, A. Yamaguchi, T. Fujishima, and H. Ohta, "Vertical GaN-based trench gate metal oxide semiconductor fieldeffect transistors on GaN bulk substrates," Appl. Phys. Exp., vol. 1, no. 1, p. 011105, Jan 2008.

[3] D. Ji, M. A. Laurent, A. Agarwal, W. Li, S. Mandal, S. Keller, and S. Chowdhury, "Normally OFF Trench CAVET With Active Mg-Doped $\mathrm{GaN}$ as Current Blocking Layer." IEEE Trans. Electron Devices, vol. 64, no. 3, pp 805-808, Mar 2017.

[4] C. Gupta, S. H. Chan, C. Lund, A. Agarwal, O. Koksaldi, J. Liu, Y. Enatsu, S. Keller, and U. K. Mishra, "Comparing electrical performance of GaN trench-gate MOSFETs with a-plane (1120) and m-plane (1100) sidewall channels," Appl. Phys. Exp., vol. 9, no. 12, pp. 121001-1- 121001-3, Nov 2016.

[5] C. Gupta, A. Agarwal, S. H. Chan, O. S. Koksaldi, S. Keller and U.K. Mishra, "1 kV field plated in-situ Oxide, GaN interlayer based vertical trench MOSFET (OG-FET)", in Proc. 75th Annu. Device Res. Conf. (DRC), Jun 2017, pp. 1-2.

[6] C. Gupta, C. Lund, S. H. Chan, A. Agarwal, J. Liu, Y. Enatsu, S. Keller and U.K. Mishra, "In-situ Oxide, GaN interlayer based vertical trench MOSFET (OG-FET) on bulk GaN substrates", IEEE Electron Device Lett., vol. 38, no. 3, pp. 353-356, Mar 2017.

[7] M. Sun, Y. Zhang, X. Gao and T. Palacios, "High performance GaN vertical fin power transistors on bulk GaN substrate", IEEE Electron Device Lett., vol. 38, no. 4, pp. 509-512, Apr 2017

[8] T. Oka, Y. Ueno, T. Ina, and K. Hasegawa, "Vertical GaN-based trench metal oxide semiconductor field effect transistors on a freestanding GaN substrate with blocking voltage of $1.6 \mathrm{kV}$," Appl. Phys. Exp., vol. 7, no. 2, p. 021002, 2014.

[9] T. Oka, T. Ina, Y. Ueno and J. Nishii, “ $1.8 \mathrm{~m} \Omega \cdot \mathrm{cm}^{2}$ vertical GaNbased trench metal-oxide-semiconductor field-effect transistors on a freestanding GaN substrate for 1.2-kV-class operation," Appl. Phys. Exp., vol. 8, no. 5, pp. 054101-1-054101-3, May 2015.

[10] T. Oka, T. Ina, Y. Ueno, and J. Nishii, "Over 10 a operation with switching characteristics of $1.2 \mathrm{kV}$-class vertical GaN trench MOSFETs on a bulk GaN substrate," in Proc. 28th Int. Symp. Power Semiconductor Devices ICs (ISPSD), Jun 2016, pp. 459-462.

[11] R. Li, Y. Cao, M. Chen and R. Chu, “600V/1.7 Normally-Off GaN Vertical Trench Metal-Oxide-Semiconductor Field-Effect Transistor", IEEE Electron Device Lett., vol. 37, no. 11, pp.1466-1469, Nov 2016.

[12] H. Nie, Q. Diduck, B. Alvarez, A. P. Edwards, B. M. Kayes, M. Zhang, G. Ye, T. Prunty, D. Bour and I. C. Kizilyalli, " $1.5-\mathrm{kV}$ and 2.2-m $\Omega . \mathrm{cm}^{2}$ vertical GaN transistors on bulk-GaN substrates," IEEE Electron Device Lett., vol. 35, no. 9, pp. 939-941, Sep 2014

[13] D. Shibata, R. Kajitani, M. Ogawa, K. Tanaka, S. Tamura, T. Hatsuda, M. Ishida, and T. Ueda, " $1.7 \mathrm{kV} / 1.0 \mathrm{~m} \Omega \cdot \mathrm{cm}^{2}$ normally-off vertical $\mathrm{GaN}$ transistor on $\mathrm{GaN}$ substrate with regrown $\mathrm{p}-\mathrm{GaN} / \mathrm{AlGaN} / \mathrm{GaN}$ semipolar gate structure," in IEDM Tech. Dig., 2016, pp. 10.1.110.1.4.

[14] X. Zhang, X. Zou, X. Lu, C. W. Tang, and K. M. Lau, "Fully- and Quasi-Vertical GaN-on-Si p-i-n Diodes: High Performance and Comprehensive Comparison," IEEE Trans. Electron Devices, vol. 64, no. 3, pp. 809-815, Mar 2017.

[15] X. Zou, X. Zhang, X. Lu, C. W. Tang, and K. M. Lau, "Fully Vertical GaN p-i-n Diodes Using GaN-on-Si Epilayers," IEEE Electron Device Lett., vol. 37, no. 5, pp. 636-639, May 2016.

[16] X. Zou, X. Zhang, X. Lu, C. W. Tang, and K. M. Lau, "Breakdown Ruggedness of Quasi-Vertical GaN-Based p-i-n Diodes on $\mathrm{Si}$ Substrates," IEEE Electron Device Lett., vol. 37, no. 9, pp. 11581161, Sep 2016.

[17] S. Mase, Y. Urayama, T. Hamada, J. J. Freedsman, and T. Egawa, "Novel fully vertical GaN p-n diode on $\mathrm{Si}$ substrate grown by metalorganic chemical vapor deposition," Appl. Phys. Exp., vol. 9, no. 11, p. 111005 , Nov 2016.

[18] Y. Zhang, M. Sun, D. Piedra, M. Azize, X. Zhang, T. Fujishima, and T. Palacios, "GaN-on-Si Vertical Schottky and p-n Diodes," IEEE Electron Device Lett., vol. 35, no. 6, pp. 618-620, Jun 2014.

[19] Y. Zhang, D. Piedra, M. Sun, J. Hennig, A. Dadgar, L. Yu, and T. Palacios, "High-Performance $500 \mathrm{~V}$ Quasi- and Fully- Vertical GaN-
on-Si pn Diodes," IEEE Electron Device Lett., vol. 38, no. 2, pp. 248 251, Feb 2017.

[20] R. A. Khadar, C. Liu, and E. Matioli, "820 V GaN-on-Si QuasiVertical PiN Diodes with BFOM of $2.0 \mathrm{GW} / \mathrm{cm}^{2}$," IEEE Electron Device Lett., vol. 39, no. 3, pp. 401-404, Mar 2018.

[21] C. Liu, R. A. Khadar, and E. Matioli, "GaN-on-Si Quasi-Vertical Power MOSFETs," IEEE Electron Device Lett., vol. 39, no. 1, pp. 7174, Jan 2018.

[22] MKodama, M. Sugimoto, E. Hayashi, N. Soejima, O. Ishiguro, M. Kanechika, K. Itoh, H. Ueda, T. Uesugi, and T. Kachi, "GaN-Based Trench Gate Metal Oxide Semiconductor Field-Effect Transistor Fabricated with Novel Wet Etching," Appl. Phys. Exp., vol. 1, no. 2, pp. 021104-1 - 021104-3, Feb 2008.

[23] D. Han, C. Oh, H. Kim, J. Shim, K. Kim, and D. Shin, "Conduction Mechanisms of Leakage Currents in InGaN/GaN-Based LightEmitting Diodes", IEEE Trans. Electron Devices, vol. 62, no. 2, pp. 587-592, Feb 2015

(C) 2018 IEEE. Personal use of this material is permitted. Permission from IEEE must be obtained for all other uses, in any current or future media, including reprinting/republishing this material for advertising or promotional purposes, creating new collective works, for resale or redistribution to servers or lists, or reuse of any copyrighted component of this work in other works. 